\title{
A Pilot Study on Hepatobiliary Scintigraphy to Monitor Regional Liver Function in ${ }^{90}$ Y Radioembolization
}

\author{
Sandra van der Velden ${ }^{1,2}$, Manon N.G.J.A. Braat ${ }^{1}$, Tim A. Labeur ${ }^{3,4}$, Mike V. Scholten ${ }^{1}$, Otto M. van Delden ${ }^{3}$, \\ Roelof J. Bennink ${ }^{3}$, Hugo W.A.M. de Jong ${ }^{1}$, and Marnix G.E.H. Lam ${ }^{1}$ \\ ${ }^{I}$ Radiology and Nuclear Medicine, University Medical Center Utrecht, Utrecht, The Netherlands; ${ }^{2}$ Image Sciences Institute, \\ University Medical Center Utrecht, Utrecht University, Utrecht, The Netherlands; ${ }^{3}$ Department of Radiology and Nuclear Medicine, \\ Amsterdam University Medical Centers, University of Amsterdam, Amsterdam, The Netherlands; and ${ }^{4}$ Department of Medical \\ Oncology, Amsterdam University Medical Centers, University of Amsterdam, Amsterdam, The Netherlands
}

Radioembolization is increasingly used as a bridge to resection (i.e., radiation lobectomy). It combines ipsilateral tumor control with the induction of contralateral hypertrophy to facilitate lobar resection. The aim of this pilot study was to investigate the complementary value of hepatobiliary scintigraphy (HBS) before and after radioembolization in the assessment of the future remnant liver. Methods: Consecutive patients with liver tumors who underwent HBS before and after ${ }^{90} \mathrm{Y}$ radioembolization were included. Regional (treated/nontreated) and whole liver function and volume were determined on HBS and CT. Changes in regional liver function and volume were correlated with the functional liver absorbed doses, determined on ${ }^{90} \mathrm{Y}$ PET/CT. In addition, the correlation between liver volume and function change was evaluated. Results: Thirteen patients (10 hepatocellular carcinoma, 3 metastatic colorectal carcinoma) were included. Liver function of the treated part declined after radioembolization (HBS-pre, $4.0 \% / \mathrm{min} / \mathrm{m}^{2}$; HBS-post, $1.9 \%$ / $\mathrm{min} / \mathrm{m}^{2} ; P=0.001$ ), whereas the function of the nontreated part increased (HBS-pre, $1.4 \% / \mathrm{min} / \mathrm{m}^{2} ;$ HBS-post, $2.8 \% / \mathrm{min} / \mathrm{m}^{2} ; P=$ 0.009 ). Likewise, treated volume decreased (pretreatment, $1,118.7$ $\mathrm{cm}^{3}$; posttreatment, $870.7 \mathrm{~cm}^{3} ; P=0.003$ ), whereas the nontreated volume increased (pretreatment, $412.7 \mathrm{~cm}^{3}$; posttreatment, 577.6 $\left.\mathrm{cm}^{3} ; P=0.005\right)$. Bland-Altman analysis revealed a large bias (29\%) between volume decrease and function decrease in the treated part and wide limits of agreement $(-7.7 \%-65.6 \%)$. The bias between volume and function change was smaller $( \pm 6.0 \%)$ in the nontreated part of the liver, but limits of agreement were still wide $(-117.9 \%-$ $106.7 \%)$. Conclusion: Radioembolization induces regional changes in liver function that are accurately detected by HBS. Limits of agreement between function and volume changes were wide, showing large individual differences. This finding indicates that HBS may have a complementary role in the management of patients for radiation lobectomy.

Key Words: hepatobiliary scintigraphy; ${ }^{99 m T c-m e b r o f e n i n ; ~ r a d i o-~}$ embolization; ${ }^{90}$ Y; radiation lobectomy

J Nucl Med 2019; 60:1430-1436

DOI: 10.2967/jnumed.118.224394

Received Dec. 3, 2018; revision accepted Feb. 20, 2019.

For correspondence or reprints contact: Sandra van der Velden, UMC Utrecht, Mail E01.132, P.O. Box 85500, 3508GA Utrecht, The Netherlands. E-mail: velden.sandra@gmail.com

Published online Apr. 6, 2019.

COPYRIGHT (C 2019 by the Society of Nuclear Medicine and Molecular Imaging.
$\mathbf{R}$ adioembolization is a treatment option for patients with inoperable liver cancer, in which radioactive microspheres (e.g., ${ }^{90}$ Y-loaded microspheres or ${ }^{166} \mathrm{Ho}$-loaded microspheres) are injected in (branches of) the hepatic artery (1). The microspheres lodge primarily in the tumor, resulting in a high tumor-absorbed radiation dose, whereas part of the microspheres will lodge in the healthy functional liver, irradiating functional liver tissue. When only part of the liver is treated, the radiation damage induces a decrease in functional liver volume in the treated part and an increase in functional liver volume in the nontreated part (2). This effect may have implications for subsequent treatments, including surgical resection of the involved part of the liver.

There has been growing interest in radioembolization as a bridge to transplantation (3) and, more recently, as a bridge to resection (4) (i.e., radiation lobectomy). Although surgery is considered curative, many patients are excluded from it because of an inadequate future-liver-remnant (FLR) volume. Radioembolization has been found to effectively induce FLR volumetric hypertrophy while simultaneously providing tumor control. As such, it may have benefits over portal vein embolization, the current standard of care in these patients (4).

Currently, management of patients for radiation lobectomy is based on clinical, laboratory, and imaging parameters (e.g., volumetry). Liver function is tested using blood markers (e.g., bilirubin and albumin) and clinically derived scores (e.g., ChildPugh and MELD). Although these give an indication of global liver function, liver function may actually be heterogeneously distributed, especially in patients with known underlying liver disease such as cirrhosis or hilar liver tumors. Hypertrophy of the FLR may therefore be insufficient for subsequent resection. A better understanding of the dose-effect relationship between radioembolization and FLR hypertrophy, in combination with a more accurate assessment of FLR in terms of functional liver reserve, may lead to better selection, planning, and monitoring of patients who have an indication for radiation lobectomy.

Quantitative total and regional liver function can be measured using hepatobiliary scintigraphy (HBS) with ${ }^{99 m} \mathrm{Tc}-$ mebrofenin (Bridatec; GE Healthcare B.V.). HBS with ${ }^{99 \mathrm{~m} T c-m e b r o f e n i n ~ b e-~}$ fore hepatectomy adequately predicts the risk of postsurgical liver failure (5-7). A cutoff of $2.69 \% / \mathrm{min} / \mathrm{m}^{2}$ (body surface area corrected ${ }^{99 m}$ Tc-mebrofenin uptake rate [cMUR, the "c" in this abbreviation stands for (body surface area) corrected]) in the FLR was reported to accurately identify patients at risk of liver failure, 
regardless of underlying liver disease, improving the presurgical work-up based on liver volumetry alone (6-8).

Two case studies $(9,10)$ reported on the feasibility of HBS to monitor regional liver function changes after radioembolization. The aim of this pilot study was to investigate the potential complementary value of regional function assessment in the management of radiation lobectomy by analyzing the correlation between regional liver function and volume changes.

\section{MATERIALS AND METHODS}

\section{Patient Selection}

All patients treated with radioembolization $(n=356)$ between April 2012 and February 2018 were reviewed. In total, 60 patients underwent HBS, of whom 17 underwent HBS before and after treatment and were hence evaluated. HBS was initially introduced as part of the TRACE study (NCT01381211 (11)). On the basis of the initial experience in that study, HBS was increasingly used in clinical routine, mostly in cirrhotic hepatocellular carcinoma patients. Two patients were excluded from the study, because the posttreatment HBS was acquired more than 4 mo after treatment (i.e., 7.5 and $17 \mathrm{mo}$ ). One patient was excluded because of additional liver-directed treatment (i.e., radiofrequency ablation) between radioembolization and posttreatment HBS, and 1 patient was excluded because of treatment with ${ }^{166} \mathrm{Ho}$-microspheres and not ${ }^{90} \mathrm{Y}$-microspheres. Hence, the data of 13 patients (11 male, 2 female) were retrospectively analyzed. Three of these patients were earlier included in a case series by Braat et al. (10). The medical ethics committee waived the need for informed consent.

Patient characteristics can be found in Table 1. Ten patients had hepatocellular carcinoma, and 3 patients had metastases from a colorectal carcinoma. The treatment intent was palliative in 5 patients, and 8 patients underwent radioembolization for downstaging or induction of hypertrophy to enable hepatectomy. Five patients were successfully resected. The other patients had either progression of disease $(n=2)$ or insufficient remnant-liver function for subsequent surgery $(n=1)$.

\section{Radioembolization}

The regular work-up included 3-phase CT or MRI and clinical or laboratory assessment of liver function. Before radioembolization treatment, all patients underwent a safety procedure. During this procedure, a scout dose of approximately $150 \mathrm{MBq}$ of ${ }^{99 \mathrm{~m}} \mathrm{Tc}$-macroaggregated albumin (TechneScan LyoMaa; Mallinckrodt Medical) was intraarterially injected. Immediately afterward, ${ }^{99 \mathrm{~m} T c-m a c r o a g-}$ gregated albumin planar scintigraphy was obtained, followed by ${ }^{99 \mathrm{~m} T c-m a c r o a g g r e g a t e d ~ a l b u m i n ~ S P E C T / C T}$. The lung shunt fraction was determined on planar scintigraphy, and SPECT/CT was used to detect extrahepatic depositions.

All patients were treated with ${ }^{90}$ Y-glass microspheres (TheraSphere; BTG International). The administered activity was calculated using the MIRD model (12). The procedure was performed according to international guidelines (13).

\section{HBS}

After intravenous administration of approximately $200 \mathrm{MBq}$ of 99mTc-mebrofenin, a dual-head $\gamma$-camera (Symbia T16; Siemens Healthcare) was positioned over the patient, with the heart and liver included in the field of view. The $\gamma$-camera was mounted with a lowenergy high-resolution collimator. The acquisition protocol consisted of 3 phases (14). First, 36 dynamic anterior and posterior images were acquired with a frame duration of $10 \mathrm{~s}$ (matrix: $128 \times 128$, energy window: $140 \mathrm{keV} \pm 7.5 \%$, zoom: 1.00). Second, a fast SPECT/CT scan was acquired (matrix: $128 \times 128$, energy window: $140 \mathrm{keV} \pm$
TABLE 1

Patient Characteristics $(n=13)$

\begin{tabular}{|c|c|}
\hline Characteristic & Data \\
\hline Age (y) & $68(50-78)$ \\
\hline \multicolumn{2}{|l|}{ Sex } \\
\hline Male & $11(85 \%)$ \\
\hline Female & $2(15 \%)$ \\
\hline \multicolumn{2}{|l|}{ Primary malignancy } \\
\hline Hepatocellular carcinoma & $10(77 \%)$ \\
\hline Metastatic colorectal carcinoma & $3(23 \%)$ \\
\hline \multicolumn{2}{|l|}{ Treatment } \\
\hline Lobar & $11(85 \%)$ \\
\hline Right & $11(85 \%)$ \\
\hline Left & $0(0 \%)$ \\
\hline Superselective & $2(15 \%)$ \\
\hline Administered ${ }^{90} \mathrm{Y}$ activity (GBq) & $2.58(1.17-7.11)$ \\
\hline $\begin{array}{l}\text { Time from }{ }^{90} Y \text { calibration to } \\
\text { treatment }(d)^{\dagger}\end{array}$ & $9(2-11)$ \\
\hline $\begin{array}{l}\text { Estimated administered } \\
\text { microspheres }\left(n \times 10^{6}\right)^{\dagger}\end{array}$ & $4.81(1.71-13.8)$ \\
\hline \multicolumn{2}{|l|}{ Average absorbed ${ }^{90} Y$ dose (Gy) } \\
\hline Treated part & $102.9(71.8-125.3)$ \\
\hline Functional parenchyma & $83.4(71.6-117.0)$ \\
\hline Tumor & $174.3(66.7-335.8)$ \\
\hline Time from HBS-pre to treatment (d) & $26(10-64)$ \\
\hline Time from treatment to HBS-post (d) & $92(58-111)$ \\
\hline Cirrhosis & $6(46 \%)$ \\
\hline Portal hypertension & $4(31 \%)$ \\
\hline \multicolumn{2}{|c|}{$\begin{array}{l}{ }^{\dagger} n=8 \text {. } \\
\text { Continuous data are median followed by range in parentheses. } \\
\text { Categoric data are frequency followed by percentage in } \\
\text { parentheses. }\end{array}$} \\
\hline
\end{tabular}

7.5\%, 64 projections, 8 s/projection, zoom: 1.45$)$. A low-dose CT scan was acquired for attenuation correction, and a diagnostic contrastenhanced CT scan was acquired for anatomic reference. In the last phase, 30 dynamic planar frames were acquired with a frame duration of $60 \mathrm{~s}$ (matrix: $128 \times 128$, energy window: $140 \mathrm{keV} \pm 7.5 \%$, zoom: $1.00)$ to evaluate biliary excretion. We will refer to HBS acquired before treatment as HBS-pre and HBS acquired after treatment as HBS-post.

\section{${ }^{90}$ Y PET/CT}

On the same day or the day after radioembolization, ${ }^{90} \mathrm{Y}$ PET/CT (Siemens Biograph mCT, time-of-flight) was performed to assess the activity distribution. The acquisition time was $15 \mathrm{~min}$ per bed position (30 min total), and consecutive bed positions overlapped by approximately 43\%. A low-dose CT scan (120 kVp, $40 \mathrm{mAs})$ was acquired for attenuation correction. PET images were reconstructed using ordinary Poisson ordered-subset expectation maximization, including resolution recovery, time-of-flight information, and correction for attenuation, randoms, and scatter. Images were reconstructed using 4 iterations and 21 subsets and were smoothed with a gaussian filter of $5 \mathrm{~mm}$ in full width at half maximum. The reconstructed voxel size was $3.9 \times 3.9 \times 4.0 \mathrm{~mm}$. 


\section{Image Analysis}

HBS. HBS was analyzed similarly to the method described by de Graaf et al. (14). A geometric mean data set was calculated from the anterior and posterior dynamic projections of the first acquisition phase. Regions of interest around the total image, liver, and cardiac blood pool were manually delineated. Subsequently, the ${ }^{99 \mathrm{~m}} \mathrm{Tc}-\mathrm{meb}$ rofenin uptake rate (MUR) expressed in \%/min was calculated according to the method of Ekman et al. (15). The liver uptake rate was divided by the body surface area (cMUR, expressed in $\% / \mathrm{min} / \mathrm{m}^{2}$ ) to correct for variability in metabolic need.

Regional liver uptake values were determined on SPECT using Simplicit ${ }^{90} Y$ software (Mirada Medical Limited). The accompanying contrast-enhanced CT scan was used for anatomic reference. When no contrast-enhanced CT scan was obtained during HBS, the lowdose CT scan used for attenuation correction was rigidly registered to a previously acquired contrast-enhanced CT scan or MRI scan $(n=2)$.

The whole liver and tumors were semiautomatically delineated on contrast-enhanced CT. The hilar and extrahepatic bile ducts were excluded from the whole liver volume of interest (VOI). After rigid registration with posttreatment ${ }^{90} \mathrm{Y}$ PET/CT, the liver VOI was manually divided into a treated part (excluding tumors) and a nontreated part, based on the ${ }^{90} \mathrm{Y}$ distribution. The function of the treated and nontreated parts was subsequently calculated as follows (7):

$$
\mathrm{cMUR}_{i}=\frac{c_{i}}{c_{\text {liver }}} \cdot \mathrm{cMUR}_{\mathrm{liver}}
$$

where $\mathrm{cMUR}_{i}$ is the liver uptake rate in VOI $i$ (i.e., treated or nontreated part), $c_{i}$ is the number of counts in VOI $i, c_{\text {liver }}$ is the number of counts in the whole liver, and $\mathrm{cMUR}_{\text {liver }}$ is the liver uptake rate calculated from the dynamic planar images. Besides liver uptake rate, volumes $\left(\mathrm{cm}^{3}\right)$ of the different VOIs were also obtained from Simplicit ${ }^{90}$ Y.
${ }^{90} Y$ PET/CT. The absorbed dose to the functional liver parenchyma was calculated using Simplicit ${ }^{90} Y$ software. The ${ }^{90} \mathrm{Y}$ PET/CT images were rigidly registered to the contrast-enhanced $\mathrm{CT}$ used to analyze HBS-pre to allow the use of identical VOIs.

\section{Statistical Analysis}

Statistical analysis was performed using the Python module Scipy, version 0.16.0 (Python Software Foundation). Categoric variables were described as frequencies and percentages, and continuous data were expressed as medians and ranges. Because of the limited sample size, data did not follow a normal distribution. Therefore, differences between groups were tested with the nonparametric Wilcoxon signedrank test. Correlation between variables was tested using the Spearman correlation coefficient, $\rho$. Correspondence between measurements was analyzed using Bland-Altman plots. A $P$ value of 0.05 or less was considered significant.

\section{RESULTS}

In general, treatment-induced toxicity within 3 mo after treatment was mild. The median follow-up was 7 mo (range: 1-30 mo). Three patients died within 6 mo after treatment, of whom 2 died from radioembolization-induced liver disease (10). These 2 patients were part of the TRACE study (11), in which HBS data were analyzed after study closure. One patient died from rapid tumor progression after radioembolization treatment.

Blood markers before and after treatment, as well as their correlation with whole liver function, can be found in Table 2 . HBS whole liver function (before and after treatment) was correlated with bilirubin, albumin, aspartate aminotransferase, and international normalized ratio. When bilirubin and albumin were combined into the albumin-bilirubin score (16), the correlation was even stronger. Whole liver volume did not correlate with bilirubin, albumin, aspartate aminotransferase,

TABLE 2

Blood Markers at Baseline (HBS-Pre) and Follow-up (HBS-Post) and Their Correlation with Whole Liver Function

\begin{tabular}{|c|c|c|c|c|}
\hline \multirow[b]{2}{*}{ Blood marker } & \multicolumn{2}{|c|}{ Baseline } & \multicolumn{2}{|c|}{ Follow-up } \\
\hline & Data & $\rho$ & Data & $\rho$ \\
\hline Bilirubin $(\mu \mathrm{mol} / \mathrm{L})^{*}$ & $9(5-31)$ & $-0.73^{\dagger}$ & $10(5-164)$ & $-0.64^{\dagger}$ \\
\hline Albumin (g/L) & $39.6(30.2-46.1)$ & $0.63^{\dagger}$ & $38.7(20.2-45.0)$ & $0.80^{\dagger}$ \\
\hline AST (U/L) & $52(22-313)$ & $-0.68^{\dagger}$ & $51(24-403)$ & $-0.84^{\dagger}$ \\
\hline ALT (U/L) & $45(12-113)$ & -0.53 & $36(8-232)$ & $-0.73^{\dagger}$ \\
\hline GGT (U/L) & $108(66-386)$ & -0.26 & $204(66-804)$ & 0.26 \\
\hline ALP (U/L) & $142(62-199)$ & $-0.7^{\dagger}$ & $176(73-347)$ & -0.19 \\
\hline $\mathrm{INR}^{\ddagger}$ & $1.03(0.82-1.40)$ & $-0.58^{\dagger}$ & $1.06(0.84-1.74)$ & $-0.51^{\dagger}$ \\
\hline ALBI score* & $-2.83(-3.21$ to -1.58$)$ & $-0.75^{\dagger}$ & $-2.60(-3.13$ to -0.55$)$ & $-0.85^{\dagger}$ \\
\hline Grade 1 & $10(77 \%)$ & - & $6(50 \%)$ & - \\
\hline Grade 2 & $3(23 \%)$ & - & $3(25 \%)$ & - \\
\hline Grade 3 & $0(0 \%)$ & - & $3(25 \%)$ & - \\
\hline \multicolumn{5}{|c|}{$\begin{array}{l}{ }^{*} n=12 \text { (follow-up). } \\
{ }^{\dagger} P<0.05 . \\
{ }^{ \pm} n=12 \text { (baseline and follow-up). } \\
\text { AST = aspartate aminotransferase; ALT = alanine aminotransferase; GGT = y-glutamyltransferase; ALP = alkaline phosphatase; } \\
\text { NR = international normalized ratio; ALBI = albumin-bilirubin. } \\
\text { Continuous data are median followed by range in parentheses. Categoric data are frequency followed by percentage in parentheses. }\end{array}$} \\
\hline
\end{tabular}



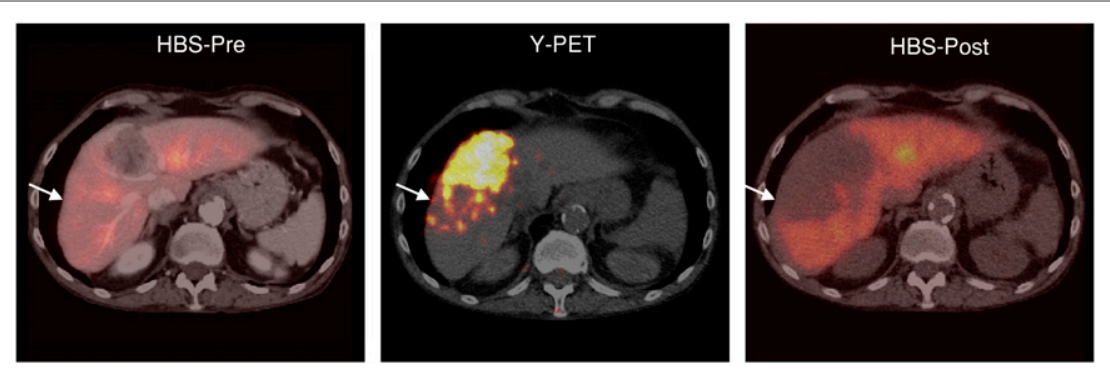

FIGURE 1. Example of regional liver function decline after ${ }^{90} Y$ radioembolization. Part of functional liver parenchyma (arrows) received a high absorbed dose of ${ }^{90} \mathrm{Y}$ (103 Gy to functional liver, 231 Gy to tumor). This was reflected on HBS-post, where that particular part of functional liver lost most of its function (HBS-pre, $2.4 \% / \mathrm{min} / \mathrm{m}^{2}$; HBS-post, $0.6 \% / \mathrm{min} / \mathrm{m}^{2}$ ).

international normalized ratio, or any other blood value at baseline or follow-up.

HBS whole liver and regional (treated/nontreated) liver function and volume at baseline and follow-up for each patient can be found in Supplemental Table 1 (supplemental materials are available at http://jnm.snmjournals.org). Overall, the function of the treated part declined after radioembolization $(P=0.001)$, whereas the function of the nontreated part increased $(P=0.009)$ (Figs. 1, $2 \mathrm{~A}$, and $3 \mathrm{~A})$. The increase in function of the nontreated part did not fully compensate for the decline in function of the treated part, as reflected by the decrease in whole liver function seen in most patients $(P=0.009)$. In only 1 patient did whole liver function increase after treatment, mainly because of a large increase in liver function in the nontreated part $(+98.4 \%)$, whereas the function of the treated part showed only a minor decline $(-5.6 \%)$. In 2 patients, liver function declined in the nontreated part of the liver. One of these patients had limited liver function at baseline (patient 3, Supplemental Table 1) and died 4 mo after treatment as a result of definite radioembolization-induced liver disease (10). The other patient had massive tumor progression in both the treated and nontreated parts of the liver and died 5 mo after treatment.

In most patients $(n=12)$, the treated volume decreased $(P=0.003)$ whereas the nontreated volume increased $(P=$ 0.005) (Figs. 2B, 3B, and 4). Whole liver volume, however, did not change significantly after radioembolization $(P=$ 0.600). For 2 patients, both with cirrhotic livers, the volume of the nontreated part decreased after radioembolization. One of these patients also had a decrease in liver function and died of radioembolizationinduced liver disease 4 mo after treatment, as described above. The other patient had a slight increase of function in the nontreated part $(+29.2 \%)$ but died 4 mo after treatment because of hepatic failure (probably radioembolization-induced liver disease, patient 2, Supplemental Table 1) (10).

No correlation was found between the absorbed dose in the treated functional liver tissue and the absolute function change $\left(\mathrm{cMUR}_{\text {post }}-\mathrm{cMUR}_{\text {pre }}\right)$ in the treated functional liver tissue $(\rho=$ $-0.31, P=0.310$ ), nor was a correlation found between absorbed dose and volume change $(\rho=0.09, P=0.768)$. However, the 3 patients who received the highest absorbed dose (average absorbed dose $>104.5 \mathrm{~Gy}$ ) showed a larger function decline (cMUR change $<-3.8 \% / \mathrm{min} / \mathrm{m}^{2}$ ) than the patients receiving a lower absorbed dose (cMUR change $\left.>-2.4 \% / \mathrm{min} / \mathrm{m}^{2}\right)(P=$ 0.007). Interestingly, these 3 patients also showed the largest function increase (cMUR change $>2.1 \% / \mathrm{min} / \mathrm{m}^{2}$ ) in the nontreated part $(P=0.011)$. No such relationships were observed for absorbed dose versus volume change.

Whole liver volume and whole liver function showed no correlation at baseline $(\rho=-0.07, P=0.817)$. Bland-Altman analysis

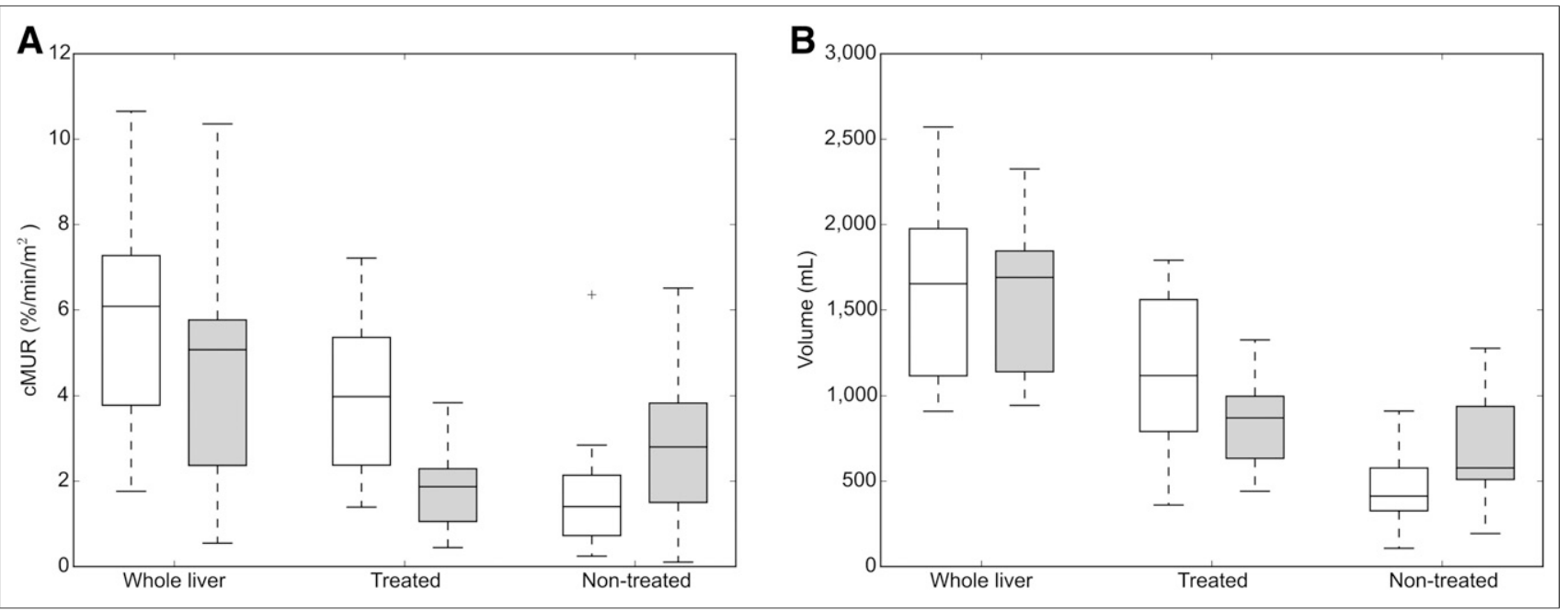

FIGURE 2. (A) Box plot of liver function obtained from HBS-pre (white) and HBS-post (gray). Whole liver function declined (HBS-pre, $6.3 \% / \mathrm{min} / \mathrm{m}^{2}$ $\left[1.8 \%-11.0 \% / \mathrm{min} / \mathrm{m}^{2}\right]$; HBS-post, $\left.5.1 \% / \mathrm{min} / \mathrm{m}^{2}\left[0.6 \%-10.6 \% / \mathrm{min} / \mathrm{m}^{2}\right] ; P=0.009\right)$. Liver function in treated part declined (HBS-pre, $4.0 \% / \mathrm{min} / \mathrm{m}^{2}[1.4 \%-$ $\left.7.2 \% / \mathrm{min} / \mathrm{m}^{2}\right]$; HBS-post, $\left.1.9 \% / \mathrm{min} / \mathrm{m}^{2}\left[0.4 \%-3.8 \% / \mathrm{min} / \mathrm{m}^{2}\right] ; P=0.001\right)$. Liver function in nontreated part increased $\left(\mathrm{HBS}-\mathrm{pre}, 1.4 \% / \mathrm{min} / \mathrm{m}^{2}\right.$ [0.2-6.4/ $\left./ \mathrm{min} / \mathrm{m}^{2}\right]$; HBS-post, $2.8 \% / \mathrm{min} / \mathrm{m}^{2}\left[0.1 \%-6.5 \% / \mathrm{min} / \mathrm{m}^{2}\right] ; P=0.009$ ). (B) Box plot of liver volume before (white) and after (gray) treatment. Whole liver volume was stable (pretreatment, $1,683.3 \mathrm{~cm}^{3}\left[983.5-3,112.5 \mathrm{~cm}^{3}\right]$; posttreatment, $\left.1,792.4 \mathrm{~cm}^{3}\left[1,012.4-3,161.2 \mathrm{~cm}^{3}\right] ; P=0.600\right)$. Healthy liver volume in treated part decreased (pretreatment, $1,118.7 \mathrm{~cm}^{3}\left[360.4-1,790.8 \mathrm{~cm}^{3}\right]$; posttreatment, $\left.870.7 \mathrm{~cm}^{3}\left[441.0-1,327.6 \mathrm{~cm}^{3}\right] ; P=0.003\right)$, whereas nontreated volume increased (pretreatment, $412.7 \mathrm{~cm}^{3}\left[107.2-910.8 \mathrm{~cm}^{3}\right]$; posttreatment, $\left.577.6 \mathrm{~cm}^{3}\left[193.5-1,278.4 \mathrm{~cm}^{3}\right] ; P=0.005\right)$. 


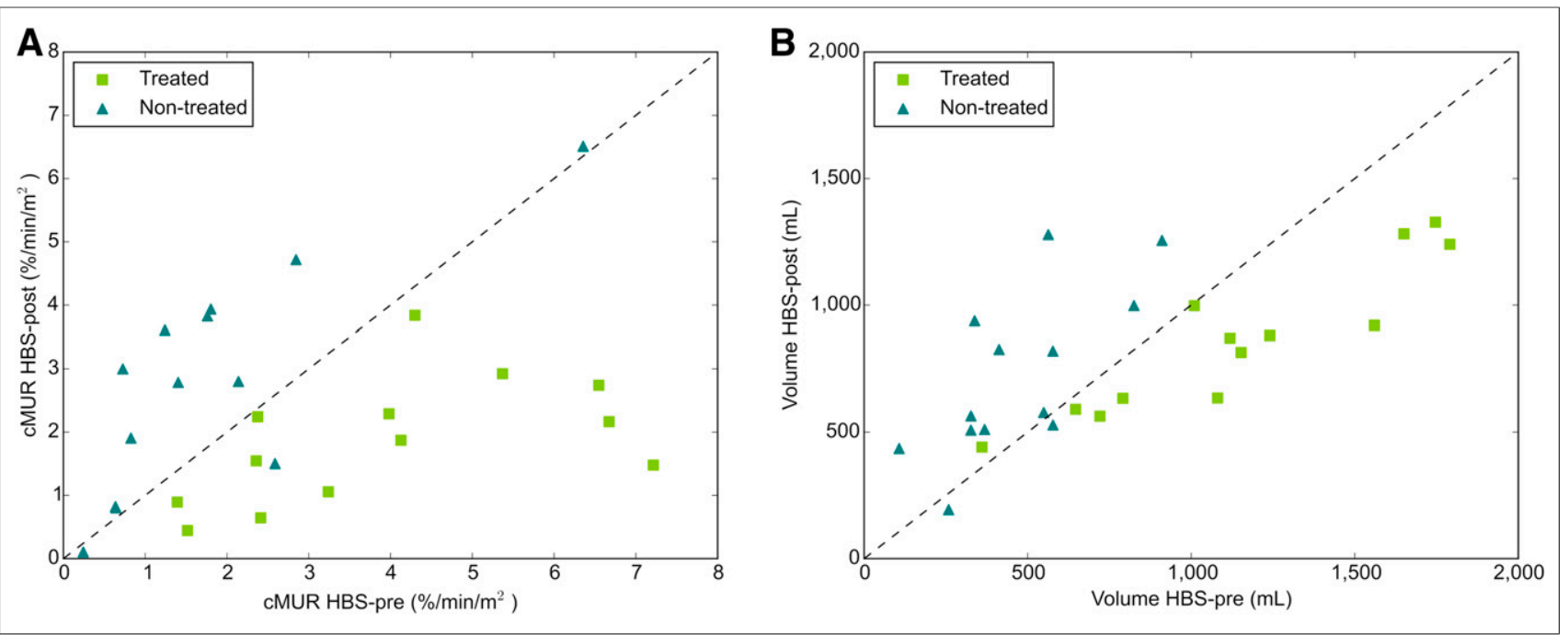

FIGURE 3. (A) Liver function obtained from HBS-pre and HBS-post and (B) liver volume before and after treatment for each individual patient. Points above dashed line indicate function/volume increase; points below dashed line indicate function/volume decline.

revealed a large bias of $29.0 \%$ and wide limits of agreement $(-7.68 \%-65.60 \%)$ for relative changes in the treated part (Fig. 5A). In the nontreated part, this bias was $-6.0 \%$ (Fig. 5B), but the limits of agreement were still wide $(-117.9 \%-106.7 \%)$. In both the treated and the nontreated parts of the liver, the individual differences were large.

Large individual differences between function and volume changes in the nontreated lobe were found: 10 of 13 patients had an increase of both function and volume, with a median relative difference between percentage function and volume increase of 61\% (range: $2 \%-134 \%$ ); 1 of 13 patients had a decrease of both, with a relative difference of $127 \%$; and 2 of 13 patients had an increase of one parameter and a decrease of the other. The relative effect in the nontreated lobe was larger for function than for volume in 10 of 13 patients.

In 2 patients, the differences between function change and volume change of the nontreated part were not concordant. One patient (patient 5; Supplemental Table 1) showed a large volume increase in the nontreated part $(+127.0 \%)$, whereas function decreased ( $\pm 41.9 \%)$. The other patient (patient 2; Supplemental Table 1 ) showed a decrease in volume $(-8.6 \%)$, but liver function in the nontreated part increased nonetheless $(+29.2 \%)$.

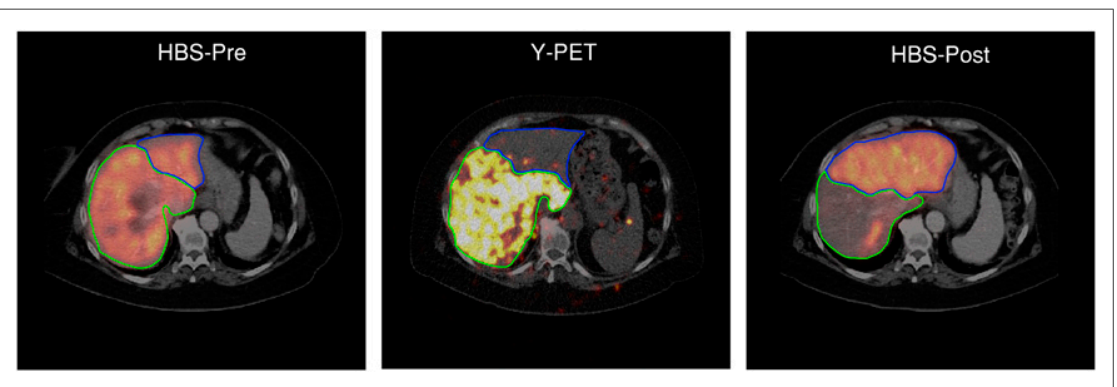

FIGURE 4. Example of volume decrease in treated part of liver $(-41 \%)$ with compensatory increase in volume of nontreated part $(+178 \%)$. Functional liver parenchyma obtained high absorbed dose of ${ }^{90} \mathrm{Y}$ (105 Gy on functional liver, 145 Gy on tumor). This particular part of liver lost most of its function (HBS-pre, $7.2 \% / \mathrm{min} / \mathrm{m}^{2}$; HBS-post, $1.5 \% / \mathrm{min} / \mathrm{m}^{2}$ ). Nontreated part increased in function (HBS-pre, $1.2 \% / \mathrm{min} / \mathrm{m}^{2}$; HBS-post, $3.6 \% / \mathrm{min} / \mathrm{m}^{2}$ ).

\section{DISCUSSION}

Lobar radioembolization induces a decrease in function and volume in the treated part of the liver and an increase in function and volume in the nontreated part. The limits of agreement between relative function and volume change were wide, reflecting large individual differences. This may indicate a complementary role for regional function assessment with HBS in the selection and treatment planning of patients undergoing radioembolization, especially in patients undergoing lobar radioembolization with the aim of inducing contralateral hypertrophy as a bridge to surgery with curative intent (i.e., radiation lobectomy) (2).

The concept of radiation lobectomy has been shown to be a feasible and effective treatment modality as a bridge to surgery in hepatocellular carcinoma patients, as an alternative to portal vein embolization (4). Although a relationship between absorbed dose to the functional liver and FLR hypertrophy in hepatocellular carcinoma patients has been shown (17), FLR hypertrophy is a poorly understood multifactorial process. From the lengthy experience with portal vein embolization, however, it is known that embolization-diverting flow toward the FLRplays a role. The embolizing properties for each radioembolization product vary considerably and, in the case of ${ }^{90} \mathrm{Y}$-glass microspheres, also depend on the interval between calibration and administration (i.e., wk 1-2 microspheres). This interval should be considered. In our study, a mix of week 1 and week 2 treatments was used for logistic reasons.

In our study, patients receiving the highest average absorbed dose to the functional liver also showed the largest function decrease in the treated part and the largest function increase in the nontreated part. In contrast, no such pattern was seen in the relationship between absorbed dose and volume change, with large individual differences between function and volume changes. With increasing attention to personalized dosimetry-based treatment planning, further investigations 


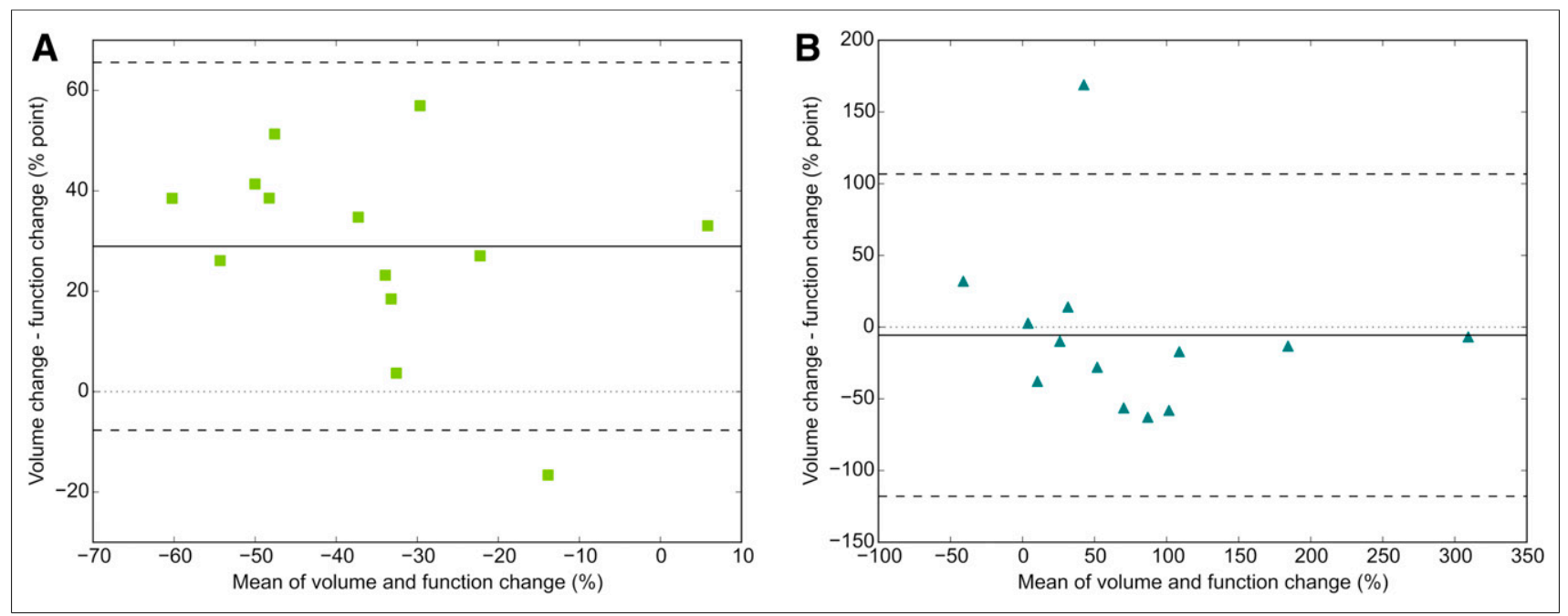

FIGURE 5. Bland-Altman plot of relative change in volume vs. relative change in function in treated part (bias, $29.0 \%$; limits of agreement, $-7.68 \%-$ $65.60 \%$ point) (A) and nontreated part (bias, $-6.0 \%$; limits of agreement, $-117.9 \%-106.7 \%$ point) (B). Solid line indicates mean; dashed lines indicate limits of agreement (mean $\pm[1.96 \times \mathrm{SD}])$.

regarding the relation between function change and absorbed dose are required and could have relevant clinical implications.

HBS with SPECT/CT allows for an accurate quantification of regional liver function. This may improve the future work-up of patients who are candidates for radioembolization, reducing the risk of hepatotoxicity. In a small case-series of 3 patients, we previously showed that discrepancies between lab values and liver function assessment using HBS may lead to dismal outcomes that potentially could have been prevented if regional HBS results had been considered (10). The suggested cMUR cutoff of $2.69 \% / \mathrm{min} /$ $\mathrm{m}^{2}$ for liver surgery (7) may be lower for lobar radioembolization, since radiation damage is a more gradual process than resection, and liver function may increase up to 12 mo after radioembolization, both in the treated and in the nontreated parts of the liver (18). Establishing the relationship between functional liver absorbed dose and functional changes is expected to lead to optimization of treatment planning by taking a prespecified FLR function into account.

Although this pilot study is the largest series to date, its main limitation is the small cohort size. Because the study was retrospective, no correlation with outcome measures (i.e., survival or hepatotoxicity) was possible, and a clear dose-effect relationship could not be established. Furthermore, liver function evaluation was performed only at $3 \mathrm{mo}$, whereas the nontreated volume increases up to 9 mo after treatment (2). It would be interesting to assess liver function and volume after a longer time after radioembolization (i.e., follow-up of 9-12 mo).

The next step toward the clinical implementation of HBS as a complementary imaging modality in radioembolization work-up would be a large, prospective validation study, in which baseline and follow-up HBS are compared with outcome measures. In addition, the relation between absorbed radiation dose and function change and the relation between FLR function and toxicity should be investigated to fully understand the potential of using HBS as an additional patient selection criterion and, possibly, a parameter for individualized dosimetry-based treatment planning.

\section{CONCLUSION}

Radioembolization induces regional changes in liver function that are accurately detected by HBS. Limits of agreement between function and volume changes after lobar radioembolization were wide, showing large individual differences. This finding indicates that HBS may have a complementary role in the management of patients for radiation lobectomy.

\section{DISCLOSURE}

This work is part of the STW-VIDI research program (project 12977), which is partly financed by the Netherlands Organization for Scientific Research. This project has received funding from the European Research Council under the European Union's Horizon 2020 research and innovation program (grant agreement 646734). Marnix G.E.H. Lam is a consultant for BTG and Terumo. The Department of Radiology and Nuclear Medicine of the UMC Utrecht receives royalties and research support from Quirem Medical/Terumo. No other potential conflict of interest relevant to this article was reported.

\section{KEY POINTS}

QUESTION: Does regional function assessment using hepatobiliary scintigraphy (HBS) have a complementary value in the management of radiation lobectomy?

PERTINENT FINDINGS: This retrospective study showed that radioembolization induces regional changes in liver function that were accurately detected by HBS. Limits of agreement between function and volume changes were wide, showing large individual differences.

IMPLICATIONS FOR PATIENT CARE: HBS may have a complementary role in the management of patients for radiation lobectomy.

\section{REFERENCES}

1. Braat AJAT, Smits MLJ, Braat MNGJA, et al. ${ }^{90} \mathrm{Y}$ hepatic radioembolization: an update on current practice and recent developments. J Nucl Med. 2015;56:10791087.

2. Vouche M, Lewandowski RJ, Atassi R, et al. Radiation lobectomy: time-dependent analysis of future liver remnant volume in unresectable liver cancer as a bridge to resection. J Hepatol. 2013;59:1029-1036.

3. Gabr A, Abouchaleh N, Ali R, et al. Comparative study of post-transplant outcomes in hepatocellular carcinoma patients treated with chemoembolization or radioembolization. Eur J Radiol. 2017;93:100-106. 
4. Gabr A, Abouchaleh N, Ali R, et al. Outcomes of surgical resection after radioembolization for hepatocellular carcinoma. J Vasc Interv Radiol. 2018;29:1502.e1-1510.e1.

5. Bennink RJ, Dinant S, Erdogan D, et al. Preoperative assessment of postoperative remnant liver function using hepatobiliary scintigraphy. J Nucl Med. 2004;45:965-971.

6. Dinant S, de Graaf W, Verwer BJ, et al. Risk assessment of posthepatectomy liver failure using hepatobiliary scintigraphy and CT volumetry. J Nucl Med. 2007;48:685-692.

7. de Graaf W, van Lienden KP, Dinant S, et al. Assessment of future remnant liver function using hepatobiliary scintigraphy in patients undergoing major liver resection. J Gastrointest Surg. 2010;14:369-378.

8. Chapelle T, Op De Beeck B, Huyghe I, et al. Future remnant liver function estimated by combining liver volumetry on magnetic resonance imaging with total liver function on ${ }^{99 \mathrm{~m}} \mathrm{Tc}$-mebrofenin hepatobiliary scintigraphy: can this tool predict post-hepatectomy liver failure? HPB. 2016;18:494-503.

9. Bennink RJ, Cieslak KP, van Delden OM, et al. Monitoring of total and regional liver function after SIRT. Front Oncol. 2014;4:152.

10. Braat MNGJA, de Jong HW, Seinstra BA. Scholten MV, van den Bosch MAAJ, Lam MGEH. Hepatobiliary scintigraphy may improve radioembolization treatment planning in HCC patients. EJNMMI Res. 2017;7:2.

11. Seinstra BA, Defreyne L, Lambert B, et al. Transarterial radioembolization versus chemoembolization for the treatment of hepatocellular carcinoma (TRACE): study protocol for a randomized controlled trial. Trials. 2012;13:144.
12. Gulec SA, Mesoloras G, Stabin M. Dosimetric techniques in ${ }^{90}$ Y-microsphere therapy of liver cancer: the MIRD equations for dose calculations. 2016:12091212 .

13. Giammarile F, Bodei L, Chiesa C, et al. EANM procedure guideline for the treatment of liver cancer and liver metastases with intra-arterial radioactive compounds. Eur J Nucl Med Mol Imaging. 2011;38:1393-1406.

14. de Graaf W, van Lienden KP, van Gulik TM, Bennink RJ. ${ }^{99 m}$ Tc-mebrofenin hepatobiliary scintigraphy with SPECT for the assessment of hepatic function and liver functional volume before partial hepatectomy. J Nucl Med. 2010;51: 229-236.

15. Ekman M, Fjälling M, Friman S, Carlson S, Volkmann R. Liver uptake function measured by IODIDA clearance rate in liver transplant patients and healthy volunteers. Nucl Med Commun. 1996;17:235-242.

16. Johnson PJ, Berhane S, Kagebayashi C, et al. Assessment of liver function in patients with hepatocellular carcinoma: a new evidence-based approach-the ALBI grade. J Clin Oncol. 2015;33:550-558.

17. Palard X, Edeline J, Rolland Y, et al. Dosimetric parameters predicting contralateral liver hypertrophy after unilobar radioembolization of hepatocellular carcinoma. Eur J Nucl Med Mol Imaging. 2018;45:392-401.

18. Theysohn JM, Ertle J, Müller S, et al. Hepatic volume changes after lobar selective internal radiation therapy (SIRT) of hepatocellular carcinoma. Clin Radiol. 2014;69:172-178. 